\title{
Approaches for quantitative study of divertor heat loads on W7-X
}

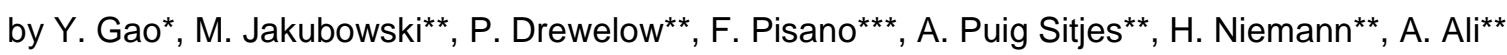 \\ and M. Rack*
}

\begin{abstract}
* Forschungszentrum Jülich GmbH, Institut für Energie- und Klimaforschung - Plasmaphysik, Partner of the Trilateral Euregio Cluster (TEC), 52425 Jülich, Germany

** Max-Planck Institut für Plasmaphysik, Association EURATOM-IPP, 17941 Greifswald, Germany

${ }^{* * *}$ Department of Electrical and Electronic Engineering, University of Cagliari, Piazza d'Armi, Cagliari 09123, Italy
\end{abstract}

\section{Abstract}

Aimed to show the fusion-reactor relevance of optimized stellarators, Wendelstein 7-X (W7-X) [1], as the world's largest stellarator (plasma volume $30 \mathrm{~m}^{3}$, major radius $5.5 \mathrm{~m}$, minor radius $0.5 \mathrm{~m}$ ), completed successfully its first experimental campaign with island divertor in December 2017. To demonstrate the capabilities of steady-state operation with reactor-relevant plasma parameters, island divertor concept is commissioned for a tolerable plasma power and particle exhaust. Pre-defined magnetic islands at the plasma boundary are cut open by the divertor plates, so that these open field lines will lead the unconfined plasmas to the graphite tiles, which are designed to allow a high power load of up to 10 $\mathrm{MW} / \mathrm{m}^{2}$.

Infrared thermography system has been developed in many fusion devices as a key diagnostic to monitor the temperature and to study the heat loads on the plasma facing components, thus to prevent them from excessive damage. W7-X are designed with superconducting coils for plasma pulses with 30 mins duration at a heating power of $10 \mathrm{MW}$. For the safety of the experimental exploration aimed at high performance long discharges, a full coverage of the field of view by infrared diagnostics for the first wall and divertor plates is essential. In this campaign, ten thermography systems were installed for the first time, with each infrared camera monitoring one half module of the machine, realized by the welldesigned wide-angle optics [2]. Figure 1 shows the field of view of the nine infrared cameras behind the immersion tubes, the temperature distributions indicate a rather symmetry target heat loads. The systems have been operated through the whole campaign with great liability, producing temperature evolution on the plasma facing components during pulses.

The infrared cameras were controlled to record the thermal emissions with a frame rate of $100 \mathrm{~Hz}$ in a wavelength range of $8-10 \mu \mathrm{m}$. The sensor array contains $768 \times 1024$ pixels, with a varied spatial resolution at different parts of the divertor surface from $\sim 3 \mathrm{~mm}$ (low iota part) to $\sim 10 \mathrm{~mm}$ (high iota tail). The exposure time has been set manually (1 to 10 $\mu \mathrm{s})$ for each discharge, depending on the heating power, to generate accurate digital levels. The Planck's law calibration under the designed optical routes, against a cavity radiator with known emissivity and temperature, has been carried out in the laboratory before the campaign. The relation between the received digital levels and the temperature has been compiled into look-up tables. The camera control and temperature conversion have been incorporated into a software platform for a real time observation of surface temperature during the experiments [3].

Except for being one of the key diagnostics for the safety of the machine [4], the divertor thermal footprints provide important inputs for the study of the intrinsic three dimensional magnetic topology and the heat transport inside the boundary islands. A quantitative analysis and comparison of the divertor temperature in different machine modules are required, as a fundamental prerequisite for the task of target heat loads symmetrisation. Due to the fivefold modular symmetry of the machine, the geometry of the ten half modules (including one horizontal and one vertical targets) are designed ideally to be identical, so that an equally distributed energy deposition onto each module could be expected, with optimized error field corrections, to support the steady-state operations.

In this work, approaches for quantitative analysis of divertor heat loads will be presented. The methods project the divertor targets of ten half modules to two identical planes (one for horizontal target and one for vertical target). Such that a directly quantitative comparison of the temperature at different targets is realized. Since each infrared camera monitoring through differed optical routes, a careful correction of optical distortions has been made specifically for each camera, as well as a mapping of camera pixels to Cartesian coordinates [5]. The method in this paper project the temperature data at the camera pixels inside the region of all divertor surfaces to two dimensional planes for quantitative studies. A grid is generated for each plane, and interpolated with real experimental temperature data, using a combination of linear and nearest interpolation methods. With the grid interpolation at identical positions for all the targets, the scattered feature of pixels for different cameras are eliminated. Figure 2 shows the average temperature maps on the horizontal and vertical targets from all divertor modules after a typical plasma discharge under the standard magnetic configuration.

Heat fluxes perpendicular to the target surfaces are calculated from the recorded time evolution of temperature on the surfaces, using THEODOR code [6], which solves the two dimensional heat diffusion equation using a numerical discretization of forward time centered space method. For a three dimensional heat fluxes as well as their radial profiles, multiple line interpolations are implemented within divertor fingers ( $50 \mathrm{~mm}$ width in toroidal direction), since a gap of thermal insulation is designed in between nearby fingers. Figure 3 shows an example of the calculated heat fluxes within one finger. 
In summary, this work reports the fundamental approaches developed for quantitative analysis of divertor heat loads on W7-X. A short introduction of W7-X and island divertor is presented. The fully covered thermography systems in W7-X have been operated successfully for the first time, through the whole experimental campaign in 2017. The set-up of the infrared diagnostics are shown, as well as the importance of thermography for both machine safety and scientific researches. Approaches for quantitative analysis will be detailed, including a mapping from camera pixels to two dimensional plane, and the heat fluxes calculation realized by a multi-line interpolation method. These tools would be utilized for various scientific studies.

\section{REFERENCES}

[1] Sunn Pedersen T, et al. Physics of Plasmas, 24, 055503 (2017).

[2] Drewelow P, et al. International Conference on Plasma Surface Interactions in Controlled Fusion Devices, (2018).

[3] Puig Sitjes A, et al. Review of Scientific Instruments, accepted.

[4] Ali A, et al. Physica Scripta, T170 014074 (6pp) (2017).

[5] Pisano F, et al. Review of Scientific Instruments, in prep. (2017).

[6] Herrmann A, et al. Plasma Physics and Controlled Fusion 37(1), 17 (1995).

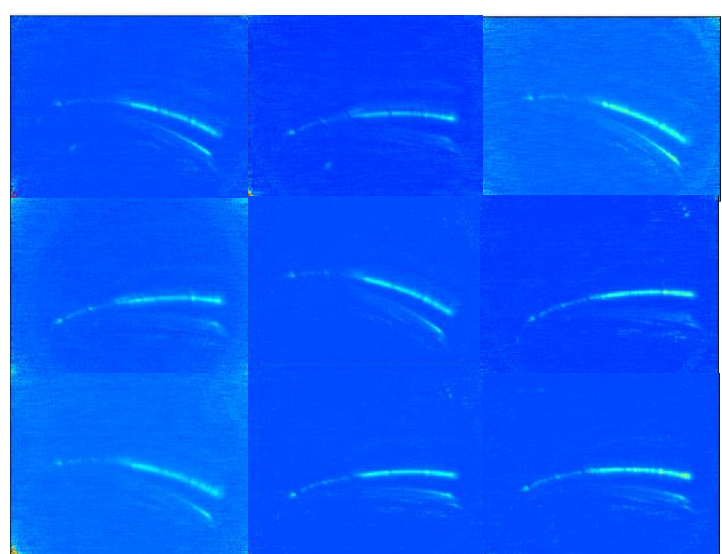

Figure 1. Field of view of the nine immersion tube infrared cameras monitoring at different divertor modules on W7$X$. It shows a typical temperature rise after a plasma discharge. The target heat loads are quasi-symmetry in this example, given an optimized error field correction.
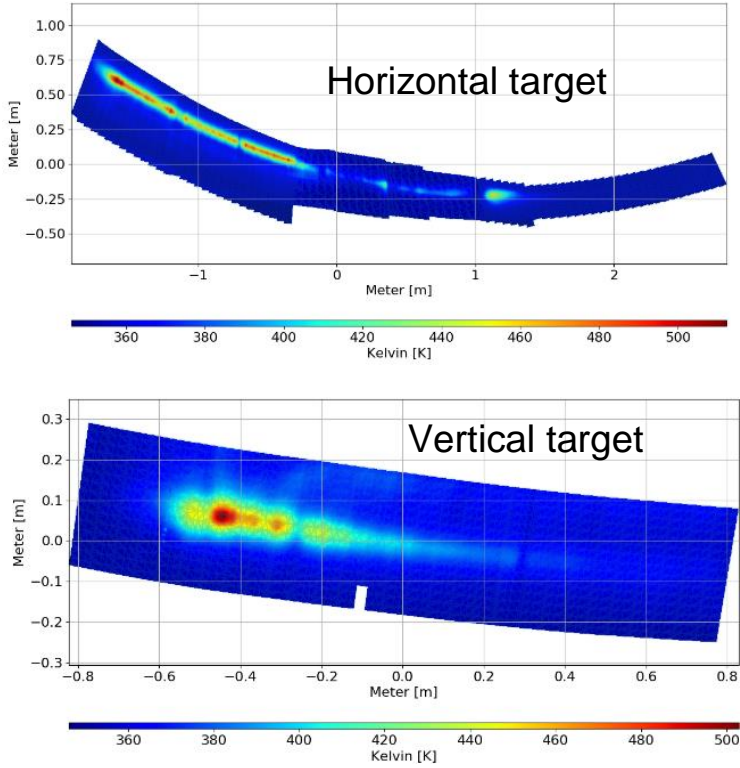

Figure 2. Averaged temperature on horizontal and vertical targets after a typical plasma discharge.
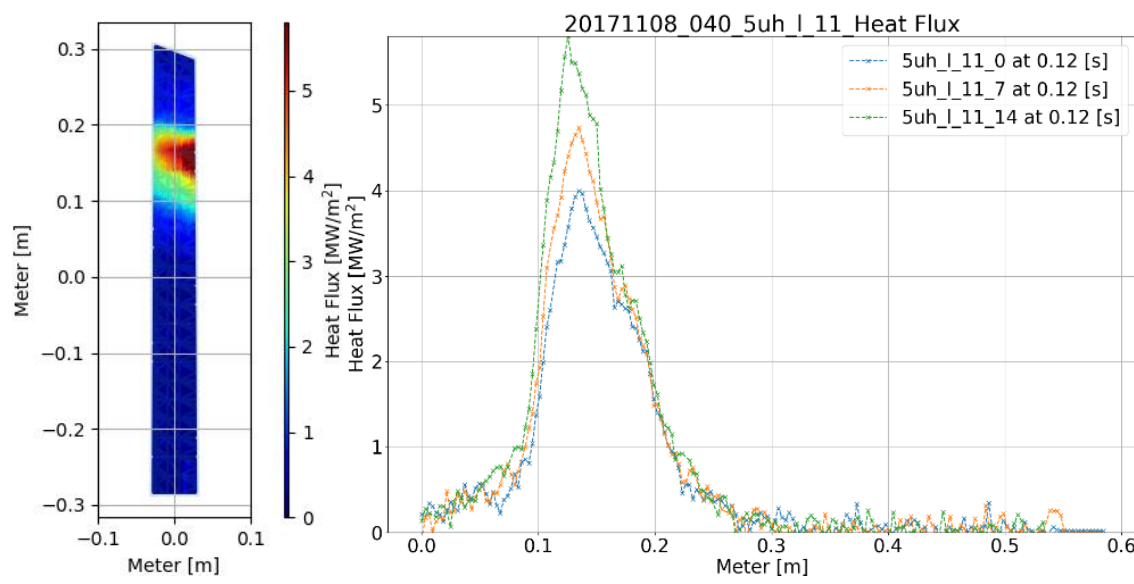

Figure 3. Left: The three dimensional heat fluxes within a typical divertor finger. Right: three heat flux profiles along the surface at the same time, with the line positions corresponds to the most left, the middle and the most right positions of the finger. 